\title{
Nonclassical joint distributions and Bell measurements
}

\author{
Elisa Masa, Laura Ares, and Alfredo Lui,* \\ Departamento de Óptica, Facultad de Ciencias Físicas, Universidad Complutense, 28040 Madrid, Spain
}

(Dated: September 12, 2019)

\begin{abstract}
Derivation and experimental violation of Bell-like inequalities involve the measurement of incompatible observables. Simple complementarity forbids the existence of such joint probability distribution. Moreover, the measurement of incompatible observables requires different experimental procedures, which no necessarily must share a common joint statistics. In this work, we avoid these difficulties by proposing a joint simultaneous measurement experiment. We can obtain the exact individual statistics of all the observables involved in the Bell inequalities after a suitable data inversion. A lack of positivity or any other pathology of the so retrieved joint distribution is then a signature of nonclassical behavior.
\end{abstract}

PACS numbers: 42.25.-p Wave optics 42.50.Dv Quantum state engineering and measurements 03.65.Ud Entanglement and quantum nonlocality 003.65.Ta Foundations of quantum mechanics; measurement theory, 42.50.Xa Optical tests of quantum theory

\section{INTRODUCTION}

Bell-like tests provide a powerful tool for testing, revealing and developing fundamental concepts at the very heart of quantum physics, involving quite basic ideas such as entanglement, reality, nonlocality, and even free will [1 [3]. Violations of the Bell inequalities provide a very valuable insight in our knowledge of nature.

A key point of Bell inequalities is that their derivation and experimental violation involve the measurement and joint statistics of incompatible observables. This is a rather interesting point since it has been shown that Bell inequalities can be derived just from the simple hypothesis of the existence of a joint probability distribution for the observables involved, without resorting to realism or nonlocality [4 8 ]. Therefore, from the very beginning, complementarity forbids the existence of such joint probability distribution, so the violation of Bell inequalities might not be so surprising after all. Alternatively, the measurement of incompatible observables requires different experimental procedures, that no necessarily must share a common joint statistics. This is a kind of practical contextuality, so to speak [8 15]. Thus, this complentarity issue really obscures the real meaning and consequences of the Bell inequalities.

In this work we propose an alternative approach that may avoid these difficulties by proposing a joint simultaneous measurement so designed that its statistics provides the exact statistics of all the observables involved in the Bell inequalities after a suitable data inversion 16 21]. In classical physics such data inversion always provides the actual joint probability distribution. Thus, lack of positivity or any other pathology of the so retrieved

\footnotetext{
*Electronic address:

URL: http://www.ucm.es/info/gioq

alluis@fis.ucm.es
}

joint distribution should be naturally linked to the violation of the Bell inequalities [4]. The point is that in this case such result would not be obscured nor diminished by complementarity or practual contextuality problems, since all observables measured would be compatible and measured in one and the same experimental arrangement.

\section{METHOD}

\section{A. System and joint measurement}

As usual, our system will be made of two subsystems $A$ and $B$, both described by a two-dimensional Hilbert space, which may be the case for example of the polarization of two photons in two distinguishable field modes.

We consider arbitrary observables defined separately in each subsystem $A, B$. These are $X, Y$ in the subsystem $A$, and $U, V$ in the subsystem $B$. All them will be described by positive operator-valued measures (POVMs) of the form

$$
\Delta_{W}(w)=\frac{1}{2}\left(\sigma_{0}+w \boldsymbol{S}_{W} \cdot \boldsymbol{\sigma}\right)
$$

for $W=X, Y, U, V$, with $w=x, y, u, v= \pm 1$ the two only values allowed for each observable, being $\boldsymbol{\sigma}$ the Pauli matrices, and $\sigma_{0}$ is the identity. $\boldsymbol{S}_{W}$ are real vectors with $\left|\boldsymbol{S}_{W}\right| \leq 1$, so that $\Delta_{W}^{\dagger}(w)=\Delta_{W}(w)$ and $\Delta_{W}(w)>0$.

For each subsystem $A, B$ we consider a joint measurement of the corresponding two observables, described by the most general POVMs for dichotomic observables in a two-dimensional space

$$
\begin{aligned}
& \tilde{\Delta}_{A}(x, y)=\frac{1}{4}\left[\sigma_{0}+\tilde{\boldsymbol{S}}_{A}(x, y) \cdot \boldsymbol{\sigma}\right], \\
& \tilde{\Delta}_{B}(u, v)=\frac{1}{4}\left[\sigma_{0}+\tilde{\boldsymbol{S}}_{B}(u, v) \cdot \boldsymbol{\sigma}\right],
\end{aligned}
$$


with

$$
\begin{gathered}
\tilde{\boldsymbol{S}}_{A}(x, y)=x \gamma_{X} \boldsymbol{S}_{X}+y \gamma_{Y} \boldsymbol{S}_{Y}+x y \gamma_{X Y} \boldsymbol{S}_{X Y} \\
\tilde{\boldsymbol{S}}_{B}(u, v)=u \gamma_{U} \boldsymbol{S}_{U}+v \gamma_{V} \boldsymbol{S}_{V}+u v \gamma_{U V} \boldsymbol{S}_{U V}
\end{gathered}
$$

where $\gamma_{X, Y, U, V}$ are real factors expressing the accuracy in the observation of each observable, and $\gamma_{X Y, U V}$ additional factors not related to the individual statistics but with information provided by the measurement beyond $X, Y$ and $U, V$. In any case, the $\gamma$ factor must be always chosen to ensure that $\left|\tilde{\boldsymbol{S}}_{A}(x, y)\right| \leq 1,\left|\tilde{\boldsymbol{S}}_{B}(u, v)\right| \leq 1$ so that $\tilde{\Delta}_{A, B}^{\dagger}=\tilde{\Delta}_{A, B}$ and $\tilde{\Delta}_{A, B}>0$.

The complete measurement is then described by the POVM product

$$
\tilde{\Delta}_{A}(x, y) \otimes \tilde{\Delta}_{B}(u, v),
$$

leading to a jointly observed statistics

$$
\tilde{p}(x, y, u, v)=\operatorname{tr}\left[\rho \tilde{\Delta}_{A}(x, y) \otimes \tilde{\Delta}_{B}(u, v)\right],
$$

where $\rho$ is the density matrix of the complete system.

\section{B. Inversion procedure}

The POVMs $\tilde{\Delta}_{A}(x, y)$ and $\tilde{\Delta}_{B}(u, v)$ are assumed to provide complete information about the corresponding observables $X, Y$ and $U, V$, respectively, so that the exact statistics of each $\Delta_{W}$ is contained in the corresponding marginal $\tilde{\Delta}_{W}$. For example, for $W=X$ we have the marginal POVM

$$
\tilde{\Delta}_{X}(x)=\sum_{y= \pm 1} \tilde{\Delta}_{A}(x, y)
$$

leading to

$$
\tilde{\Delta}_{X}(x)=\frac{1}{2}\left(\sigma_{0}+x \gamma_{X} \boldsymbol{S}_{X} \cdot \boldsymbol{\sigma}\right),
$$

and equivalently for all the other POVMs.

Thus, we assume that for each $W$ there are stateindependent functions $\mu_{W}\left(w, w^{\prime}\right)$ so that the exact $\Delta_{W}(w)$ in Eq. (1) is obtained from the marginal $\tilde{\Delta}_{W}(w)$ in Eq. (7) as

$$
\Delta_{W}(w)=\sum_{w^{\prime}= \pm 1} \mu_{W}\left(w, w^{\prime}\right) \tilde{\Delta}_{W}\left(w^{\prime}\right) .
$$

It can be easily seen that these functions are

$$
\mu_{W}\left(w, w^{\prime}\right)=\frac{1}{2}\left(1+\frac{w w^{\prime}}{\gamma_{W}}\right) .
$$

Applying the inversion procedure to the complete measurement (44) we get the family of operators $\Delta_{A}(x, y) \otimes$ $\Delta_{B}(u, v)$ where

$$
\begin{aligned}
& \Delta_{A}(x, y)=\sum_{x^{\prime}, y^{\prime}= \pm 1} \mu_{X}\left(x, x^{\prime}\right) \mu_{Y}\left(y, y^{\prime}\right) \tilde{\Delta}_{A}\left(x^{\prime}, y^{\prime}\right), \\
& \Delta_{B}(u, v)=\sum_{u^{\prime}, v^{\prime}= \pm 1} \mu_{U}\left(u, u^{\prime}\right) \mu_{V}\left(v, v^{\prime}\right) \tilde{\Delta}_{B}\left(u^{\prime}, v^{\prime}\right),
\end{aligned}
$$

leading to

$$
\begin{aligned}
& \Delta_{A}(x, y)=\frac{1}{4}\left[\sigma_{0}+\boldsymbol{S}_{A}(x, y) \cdot \boldsymbol{\sigma}\right], \\
& \Delta_{B}(u, v)=\frac{1}{4}\left[\sigma_{0}+\boldsymbol{S}_{B}(u, v) \cdot \boldsymbol{\sigma}\right],
\end{aligned}
$$

with

$$
\begin{aligned}
& \boldsymbol{S}_{A}(x, y)=x \boldsymbol{S}_{X}+y \boldsymbol{S}_{Y}+x y \frac{\gamma_{X Y}}{\gamma_{X} \gamma_{Y}} \boldsymbol{S}_{X Y}, \\
& \boldsymbol{S}_{B}(u, v)=u \boldsymbol{S}_{U}+v \boldsymbol{S}_{V}+u v \frac{\gamma_{U V}}{\gamma_{U} \gamma_{V}} \boldsymbol{S}_{U V} .
\end{aligned}
$$

Finally the inferred joint distribution is obtained as

$$
p(x, y, u, v)=\operatorname{tr}\left[\rho \Delta_{A}(x, y) \otimes \Delta_{B}(u, v)\right] .
$$

We have to stress that in classical physics this inversion procedure always leads to a proper joint probability distribution [19, 20].

\section{INVERSION AND STATISTICS FOR AN ARBITRARY STATE}

Now we apply the inversion procedure to a general case in order to analyze the possibles patologies of the joint distribution in parallel with the main features of the Bell scenario.

\section{A. System state}

For the purpose of obtaining the maximum generality, we describe the complete system state via the most general density matrix that can be always expressed as [22, 23]

$$
\begin{gathered}
\rho=\frac{1}{4}\left(\sigma_{0} \otimes \sigma_{0}+\right. \\
\left.\sum_{i=1}^{3} a_{i} \sigma_{i} \otimes \sigma_{0}+\sum_{i=1}^{3} b_{i} \sigma_{0} \otimes \sigma_{i}+\sum_{i=1}^{3} c_{i} \sigma_{i} \otimes \sigma_{i}\right),
\end{gathered}
$$

for $i=1,2,3$, where $\sigma_{i}$ are the Pauli matrices and $\boldsymbol{a}=$ $\left\{a_{i}\right\}, \boldsymbol{b}=\left\{b_{i}\right\}, \boldsymbol{c}=\left\{c_{i}\right\}$ are real vectors.

\section{B. Obtaining statistics}

For the state (14) we have that the observed joint statistics and the inferred distribution are:

$$
\begin{gathered}
\tilde{p}(x, y, u, v)= \\
\frac{1}{16}\left[1+\boldsymbol{a} \cdot \tilde{\boldsymbol{S}}_{A}(x, y)+\boldsymbol{b} \cdot \tilde{\boldsymbol{S}}_{B}(u, v)+\sum_{i=1}^{3} c_{i} \tilde{S}_{A_{i}} \tilde{S}_{B_{i}}\right]
\end{gathered}
$$


and

$$
\begin{gathered}
p(x, y, u, v)= \\
\frac{1}{16}\left[1+\boldsymbol{a} \cdot \boldsymbol{S}_{A}(x, y)+\boldsymbol{b} \cdot \boldsymbol{S}_{B}(u, v)+\sum_{i=1}^{3} c_{i} S_{A_{i}} S_{B_{i}}\right]
\end{gathered}
$$

where $\tilde{\boldsymbol{S}}_{A}, \tilde{\boldsymbol{S}}_{B}, \boldsymbol{S}_{A}$ and $\boldsymbol{S}_{B}$ are in Eqs. (3) and (12), while $S_{A_{i}}$ are the corresponding vector components.

Let us stress that there are restrictions on the modulus of $\tilde{\boldsymbol{S}}_{A, B}$ since they determine the statistics of measured observables, and also restrictions on the modulus of all $\boldsymbol{S}_{X, Y, U, V}$ since they determine the true statistics of the corresponding observables. However, there are no such restrictions of modulus for $\boldsymbol{S}_{A, B}$. And this allows for the appearance of pathologies in the inferred statistics (16) depending on the system state $\rho$.

Before showing this let us examine all marginals of this joint distribution $p(x, y, u, v)$ to properly complete the analysis. The one-observable marginals are by construction the exact ones:

$$
p_{W}(w)=\frac{1}{2}\left(1+w \boldsymbol{a} \cdot \boldsymbol{S}_{\boldsymbol{W}}\right)
$$

for $W=X, Y, w=x, y$, and

$$
p_{W}(w)=\frac{1}{2}\left(1+w \boldsymbol{b} \cdot \boldsymbol{S}_{\boldsymbol{W}}\right)
$$

for $W=U, V$. The joint $A$ and $B$ marginals are:

$$
\begin{aligned}
& p_{X, Y}(x, y)=\frac{1}{4}\left[1+\boldsymbol{a} \cdot \boldsymbol{S}_{\boldsymbol{A}}(x, y)\right], \\
& p_{U, V}(x, y)=\frac{1}{4}\left[1+\boldsymbol{b} \cdot \boldsymbol{S}_{\boldsymbol{B}}(u, v)\right] .
\end{aligned}
$$

The cross two-observable marginals:

$$
\begin{gathered}
p_{X, U}(x, u)= \\
\frac{1}{4}\left(1+u \boldsymbol{b} \cdot \boldsymbol{S}_{U}+x \boldsymbol{a} \cdot \boldsymbol{S}_{x}+x u \sum c_{i} S_{X_{i}} S_{U_{i}}\right),
\end{gathered}
$$

and equivalently for $p_{X, V}, p_{Y, U}$, and $p_{Y, V}$. At difference with Eq. (19) this is the joint statistics of two commuting observables $X$ and $U$, so it has no problem to be defined directly in terms of their common eigenvectors. Let us briefly show that actually Eq. (20) is exactly the true joint distribution for $X$ and $U$, as a further proof of the validity of our method. To this end we recall that the POVM elements associated to $X$ and $U$ are, in their corresponding Hilbert spaces,

$\Delta_{X}(x)=\frac{1}{2}\left(\sigma_{0}+x \boldsymbol{S}_{X} \cdot \boldsymbol{\sigma}\right), \quad \Delta_{U}(u)=\frac{1}{2}\left(\sigma_{0}+u \boldsymbol{S}_{U} \cdot \boldsymbol{\sigma}\right)$,

so that the joint statistics is

$$
p_{X, U}(x, u)=\operatorname{tr}\left[\rho \Delta_{X}(x) \otimes \Delta_{U}(u)\right] .
$$

For the state (14) and using the good properties of Pauli matrices under the trace we readily get that this is exactly the same in Eq. (20).

More interesting results arise for three-observable marginals such as

$$
\begin{gathered}
p_{X, U, V}(x, u, v)= \\
\frac{1}{8}\left[1+\boldsymbol{b} \cdot \boldsymbol{S}_{B}+x \boldsymbol{a} \cdot \boldsymbol{S}_{X}+x \sum_{i=1}^{3} c_{i} S_{X_{i}} S_{B_{i}}(u, v)\right] .
\end{gathered}
$$

Equivalent expressions hold for $p_{Y, U, V}, p_{X, Y, U}$, and $p_{X, Y, V}$. These are maybe the most interesting marginals for our purposes since it is the pathology of these distributions which is responsible for the violation of Bell inequalities, as we show next. This is because they are the simplest distributions containing both observables of subsystems A and B as well as two complementary observables such as $X$ and $Y$.

\section{PATHOLOGY AND VIOLATION OF BELL INEQUALITIES}

Let us show explicitly that violation of Bell-like inequalities implies that the retrieved joint distribution $p(x, y, u, v)$ takes negative values, in agreement with Fine's theorem [4]. To this end we use Bell-like inequalities expressed directly in terms of probabilities [4, 7]. This is that the existence of a legitimate probability distribution $p(x, y, u, v)$ implies the satisfaction of four double inequalities of the form, for $x, u= \pm 1$ :

$$
\begin{aligned}
0 \geq p_{X, U}(x, u) & -p_{X, V}(x, v)+p_{Y, U}(y, u)+p_{Y, V}(y, v) \\
& -p_{Y}(y)-p_{U}(u) \geq-1,
\end{aligned}
$$

and three other equivalent relations obtained by suitably permuting the observables.

We define $C$ as the nucleus of the Bell-like double inequality (24):

$$
\begin{gathered}
C=p_{X, U}(x, u)-p_{X, V}(x, v)+ \\
p_{Y, U}(y, u)+p_{Y, V}(y, v)-p_{Y}(y)-p_{U}(u),
\end{gathered}
$$

so that the inequilites are

$$
0 \geq C \geq-1
$$

Now, using the expressions (20) and (23) for the twoand three-observable marginals, it can be proved that

$$
\begin{gathered}
\Sigma=p_{X, U, V}(x, u,-v)+p_{X, U, V}(-x,-u, v) \\
+p_{Y, U, V}(y, u, v)+p_{Y, U, V}(-y,-u,-v) \\
=C+1 .
\end{gathered}
$$

Note that if there were a legitimate joint distribution then $1 \geq \Sigma \geq 0$. It must be nonnegative since it is a sum 
of probabilities and is less than or equal to one since, for all $W=X, Y$ and $w=x, y= \pm 1$ we have

$$
p_{W, U, V}(w, u, v) \leq p_{U, V}(u, v),
$$

and therefore

$$
\begin{gathered}
\Sigma \leq p_{U, V}(u,-v)+p_{U, V}(-u, v) \\
+p_{U, V}(u, v)+p_{U, V}(-u,-v)=1 .
\end{gathered}
$$

If we replace $C=\Sigma-1$ in Eq. (26), the Bell-like inequalities are equivalent to:

$$
1 \geq \Sigma \geq 0
$$

Therefore any of the violations of the Bell-like inequalities means either $\Sigma<0$ or $\Sigma>1$, which are incompatible with a legitime joint distribution.

Consequently, our scheme provides a nice and useful realization of the more abstract scenarios on the subject.

\section{CONCLUSIONS}

We have presented an alternative approach to Belllike scenarios with many fruitful possibilities. It provides a suitable arena to investigate basic quantum features such as complementarity, entanglement, nonclassicality within one and the same framework, avoiding previous assumptions that may obscure the proper elucidation of these basic features.

\section{ACKNOWLEDGMENTS}

L. A. and A. L. acknowledge financial support from Spanish Ministerio de Economía y Competitividad Project No. FIS2016-75199-P. L. A. acknowledges financial support from European Social Fund and the Spanish Ministerio de Ciencia Innovación y Universidades, Contract Grant No. BES-2017-081942.
[1] L. E. Ballentine, Quantum Mechanics (Prentice Hall, Englewood Cliffs, 1990). Chapter 20.

[2] J. S. Bell, On the Einstein Podolsky Rosen paradox, Physics 1, 195-200 (1964).

[3] R. F. Werner and M. M. Wolf, Bell inequalities and Entanglement, arXiv:quant-ph/0107093

[4] A. Fine, Hidden Variables, Joint Probability, and the Bell Inequalities, Phys. Rev. Lett 48, 291-295 (1982).

[5] A. Rivas, Incompatible Statistics and Bell-KS Theorem, arXiv:1501.04929 [quant-ph].

[6] J. A. de Barros, J. V. Kujala and G. Oas, Negative probabilities and contextuality, J. Math. Psychol. 74, 34-45 (2016).

[7] J. F. Clauser and M. A. Horne, Experimental consequences of objective local theories, Phys. Rev. D 10, 526535 (1974).

[8] W. M. Muynck and O. Abu-Zeid, On an alternative interpretation of the Bell inequalities, Phys. Lett. A 100, 485-489 (1984).

[9] A. Khrennikov, Non-Kolmogorov probability models and modified Bell's inequality, arXiv:0003.017 [quant-ph].

[10] K. Hess and W. Philipp, Bell's theorem: Critique of proofs with and without inequalities, arXiv: 0410.015 [quant-ph].

[11] A. Matzkin, Is Bell's theorem relevant to quantum mechanics. On locality and non-commuting observables, arXiv: 0802.0613 [quant-ph].

[12] T. M. Nieuwenhuizen, Is the Contextuality Loophole Fatal for the Derivation of Bell Inequalities?, Found. Phys. 41, 580 (2011).

[13] A. Khrennikov, CHSH inequality: Quantum probabilities as classical conditional probabilities, arXiv:1406.4886 [quant-ph].

[14] J. Christian, On a Surprising Oversight by John S. Bell in the Proof of his Famous Theorem, arXiv:1704.02876 [physics.gen-ph].

[15] N. N. Vorob'ev, Consistent families of measures and their extensions, Theor. Probab. Applications VII, 147 (1962).

[16] W. M. de Muynck and H. Martens, Joint measurement of incompatibles observables and ,the Bell inequalities Phys. Lett. A 142, 187-190 (1989).

[17] W. M. Muynck, Foundations of Quantum Mechanics, an Empiricist Approach, (Kluwer Academic Publishers, 2002).

[18] W. M. Muynck, Interpretations of quantum mechanics, and interpretations of violation of Bell's inequality, arXiv:quant-ph/0102066.

[19] A. Luis, Nonclassical states from the joint statistics of simultaneous measurements, http://arxiv.org/abs/1506.07680 A. Luis, Nonclassical light revealed by the joint statistics of simultaneous measurements, Opt. Lett. 41, 1789-1792 (2016).

[20] A. Luis and L. Monroy, Nonclassicality of coherent states: Entanglement of joint statistics, Phys. Rev A 96, 063802 (2017).

[21] R. Galazo, I. Bartolomé, L. Ares, and A. Luis, Classical and quantum complementarity, impossible distributions and how much quantumness is truly quantum, arXiv:1811.12636 [quant-ph].

[22] D. Girolami and G. Adesso, Quantum discord for general two-qubit states: Analytical progress, Phys. Rev. A 83, 052108 (2011).

[23] Y. Ben-Aryeh and A. Mann, Explicit constructions of all separable two-qubits density matrices and related problems for three-qubits systems, arXiv:1510.07222 [quant$\mathrm{ph}$. 